\title{
Field-dependent exciton dissociation in organic heterojunction solar cells
}

\author{
Andreas Petersen, ${ }^{1, *}$ Antti Ojala, ${ }^{2}$ Thomas Kirchartz, ${ }^{3}$ Thomas A. Wagner, ${ }^{1}$ Frank Würthner, ${ }^{4}$ and Uwe Rau ${ }^{5}$ \\ ${ }^{1}$ Robert Bosch GmbH, Robert-Bosch-Platz 1, D-70839 Gerlingen-Schillerhöhe, Germany \\ ${ }^{2}$ BASF SE, Carl-Bosch-Straße 38, D-67056 Ludwigshafen, Germany \\ ${ }^{3}$ Blackett Laboratory of Physics, Imperial College, South Kensington, London SW7 2AZ, United Kingdom \\ ${ }^{4}$ Institut für Organische Chemie and Röntgen Research Center for Complex Material Systems, Universität Würzburg, \\ Am Hubland, 97074 Würzburg, Germany \\ ${ }^{5}$ IEK5-Photovoltaik, Forschungszentrum Jülich, 52425 Jülich, Germany
}

(Received 25 September 2011; revised manuscript received 4 May 2012; published 14 June 2012)

\begin{abstract}
In organic heterojunction solar cells, the generation of free charge carriers takes place in a multistep process which involves charge transfer (CT) states, that is, bound electron-hole pairs at the interface between donor and acceptor molecules. Past efforts to model the CT-state dissociation during solar cell operation were not able to consistently reproduce the experimentally observed field and temperature dependence. This discrepancy between model and experiment was partly due to the field-dependent free charge carrier collection process, which plays an important role in the widely used bulk heterojunction cell configuration and superimposes a possible field-dependent charge carrier generation process. In order to distinguish between generation and collection of free charge carriers, we propose the planar heterojunction cell configuration as a model system to study the field-dependent charge carrier generation process in organic heterojunction solar cells. We apply this model system to check current CT-state dissociation models against experimental data. Although the models can quantitatively account for the photocurrent's dependence on the applied voltage and the device thickness, they fail to account for the virtually negligible temperature dependence of the field-dependent charge-generation process. This discrepancy is traced back to a common feature of the models: an Arrhenius-like temperature dependence, distinctive of all processes involving a thermally activated jump over an energy barrier. As a solution to the problem, we introduce an exciton dissociation model based on a field-dependent tunnel process and demonstrate its consistency with the experimental observations. Our results indicate that the current microscopic picture of the charge-generation process in organic heterojunction solar cells being limited by the CT-state dissociation process needs to be reconsidered.
\end{abstract}

DOI: 10.1103/PhysRevB.85.245208

PACS number(s): 88.40.jr, 71.35.-y, 73.61.Ph

\section{INTRODUCTION}

Organic heterojunction solar cells reach power conversion efficiencies of about $10 \%$ and are considered a low-cost alternative for sustainable energy generation. ${ }^{1,2}$ A key to further enhance the efficiency of these devices is a detailed understanding of the multistep process leading from the absorption of photons to the extraction of free charge carriers. ${ }^{3,4}$ Initially, the absorption of a photon generates an exciton, a strongly bound electron-hole pair. After the exciton has diffused to an interface between donor and acceptor molecules, the local energy offset promotes a charge transfer across the interface., ${ }^{5,6}$ The result is a charge transfer (CT) state, a Coulombically bound electron-hole pair located on adjacent donor and acceptor molecules. The subsequent dissociation of the CT-state, which has been assumed to be assisted by the cell's internal electric field, has been considered to be the ratelimiting step in the free charge carrier generation process..$^{7-11}$

Recently, the picture of the charge-generation process being limited by the CT-state dissociation was questioned. Transient absorption spectroscopy studies on different polymer/methanofullerene blends revealed that the majority of the free charge carriers were not generated via CT-states but directly by ultrafast exciton dissociation. ${ }^{12,13}$ Thus, the ratelimiting step in the free charge carrier generation process may be the dissociation of the exciton rather than the dissociation of the CT-state. ${ }^{14}$
An important difference between the two mechanisms is their temperature dependence. Because the CT-states are lower in energy than the free charge carrier pairs, the models of Onsager-Braun ${ }^{15}$ and Barker et al. ${ }^{7}$ assume the CT-state dissociation to be thermally activated. The result is an Arrhenius-like, that is, exponential temperature dependence of the CT-state dissociation, which has not been observed experimentally. ${ }^{2}$ In contrast, the exciton dissociation is an energy-releasing process in which an energy barrier that may hinder the dissociation can be tunneled. Such a tunnel process would also be compatible with the observed temperature-independent charge carrier photogeneration in polymer/methanofullerene blends. ${ }^{16}$

On a device level, the field-dependent charge photogeneration process was mainly studied using bulk heterojunction (BHJ) solar cells which featured mixed donor/acceptor (D/A) layers. ${ }^{8-11}$ In these papers, the voltage-dependent photocurrent was explained by a field-dependent CT-state dissociation process according to the Onsager-Braun (OB) formalism. ${ }^{15}$ However, none of these attempts yielded conclusive and reliable information on the CT-state dissociation process. One issue was the large discrepancy between modeled and experimental CT-state lifetimes $\tau_{\mathrm{CT}}$. While experiments determined $\tau_{\mathrm{CT}}$ to be a few nanoseconds, ${ }^{12,13,17}$ the OB model required $\tau_{\mathrm{CT}}$ to be on the order of microseconds ${ }^{8-11,18,19}$ to millisecond ${ }^{20}$ in order to reproduce the observed internal quantum efficiencies of up to $100 \% .^{21}$ Moreover, the influence of the device 
thickness could not be reproduced, ${ }^{11,22}$ and two publications on BHJ cells made of poly(3-hexyl-thiophene) (P3HT) and the fullerene derivative [6,6]-phenyl-C61 butyric-acid methylester (PCBM) reported contradictory results for the material system's zero field CT-state dissociation probability $p_{\mathrm{CT}, 0}$ of $40 \%-60 \%$ and $90 \%$, respectively. ${ }^{11,22}$

The reason for the discrepancies between model and experiment could have been the nongeminate recombination of free charge carriers. This process takes place along the extensive D/A interface of BHJ solar cells and causes a pronounced voltage dependence of the charge carrier collection efficiency which superimposes that of the CT-state dissociation. Recently, it was demonstrated that such nongeminate recombination losses, which occurred after exciton and CT-state dissociation, could explain the voltage dependence of the photocurrent of several state-of-the-art BHJ solar cells. $^{23-27}$ In such a case, the additional application of a field-dependent CT-state dissociation process has to result in overparametrization and ambiguous results.

This paper avoids the above-mentioned ambiguity by studying the field-dependent charge generation using planar heterojunction (PHJ) solar cells, whose geometrically well ordered donor and acceptor phases and the small D/A interface area result in a weak field dependence of the free charge carrier collection efficiency. We introduce and compare two field-dependent charge-generation models for PHJ solar cells. The first model is based on the assumption that the charge-generation process is limited by the CT-state dissociation, while the other treats the initial dissociation of the exciton as the limiting process. These two models are checked against experimental data of merocyanine/ $\mathrm{C}_{60} \mathrm{PHJ}$ cells whose charge-generation process, although exhibiting a distinct dependence on the applied voltage and the cell thickness, features virtually no temperature dependence. We show that our experimental data are incompatible with a key assumption of current CT-state dissociation models: a charge-generation process which is limited by the dissociation of a bound precursor state that is lower in energy than the charge-separated state.

\section{EXPERIMENT}

\section{A. Methods}

The organic solar cells used for the study of the field-dependent exciton dissociation consisted of the fullerene $\mathrm{C}_{60}$ as the acceptor material and the merocyanine dye ID583 (1-propyl-2-[2-(3,3-dimethyl-1,3-dihydroindol-2-ylidene)-ethylidene]-3-dicyanovinyl-indan-1-one) as the donor material. This D/A combination yielded an effective band gap $E_{\mathrm{eff}}=E_{\mathrm{LUMO}}$,acceptor $-E_{\mathrm{HOMO} \text {, donor }}$ (where LUMO stands for lowest unoccupied molecular orbital and HOMO stands for highest occupied molecular orbital) of $1.7 \mathrm{eV}$ and thus allowed open-circuit voltages $V_{\mathrm{oc}}$ of more than $1 \mathrm{~V}$. Figure 1 shows an overview of the layer sequence, the molecular structures, and the energy levels of the materials used in the cell stack. ${ }^{28}$ Bulk heterojunction device results for similar chromophores have been published, ${ }^{33,34}$ and it has been shown that these materials can be processed wetchemically from solution as well as by vacuum thermal
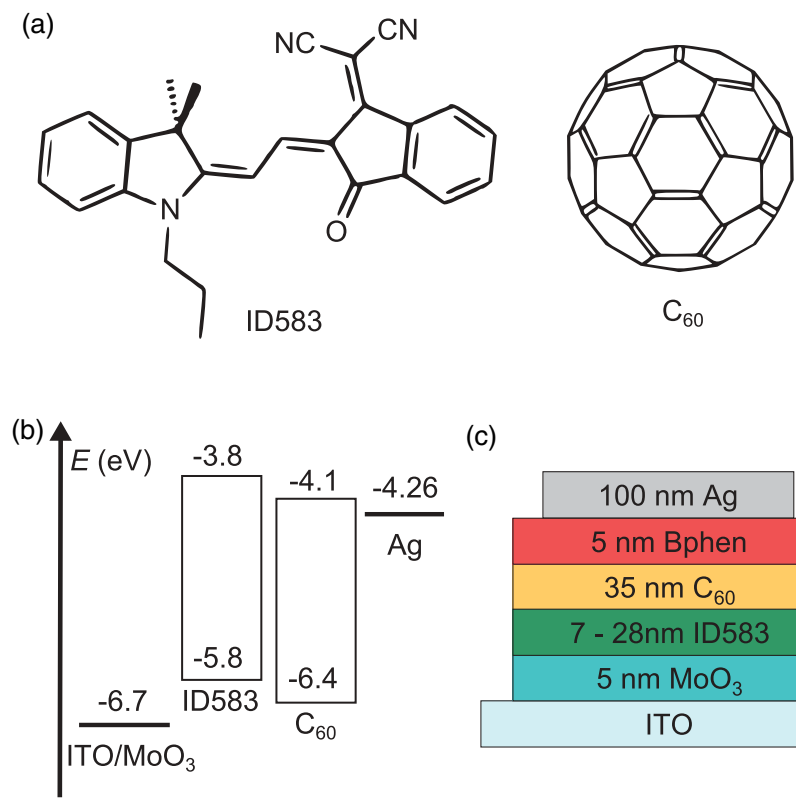

(c)

FIG. 1. (Color online) (a) Molecular structures of ID583 and $\mathrm{C}_{60}$. (b) Energy levels of the materials used in the cell stack. (c) Cell stack of the PHJ solar cells. The HOMO and the band gap of ID583 were measured by cyclic voltammetry and absorption measurements, respectively (Ref. 28). Both the HOMO (Ref. 29) and the band gap of $\mathrm{C}_{60}$ (Ref. 30) were chosen according to the literature. The work function of the ITO anode was adjusted using a thin $\mathrm{MoO}_{3}$ layer (Ref. 31) and the work function of the silver cathode was chosen according to Ref. 32. For clarity, the energy levels of the Bphen (4,7-diphenyl-1,10-phenanthroline) buffer layer are not shown.

evaporation. ${ }^{35}$ In this work thermal evaporation was used because it allowed for a simpler fabrication of well-defined multilayer structures like PHJ cells. The cells were fabricated in a vacuum chamber having a base pressure of $2 \times 10^{-6}$ mbar. The prestructured, indium tin oxide (ITO) coated glass substrates had an active cell area of $4 \mathrm{~mm}^{2}$. Prior to the layer deposition, the substrates were ex situ treated with UV ozone for $15 \mathrm{~min}$. The following layer sequence was subsequently deposited: $\mathrm{MoO}_{3}(5 \mathrm{~nm}) / \operatorname{ID} 583$ (7, 14, 21, 28 $\mathrm{nm}) / \mathrm{C}_{60}(35 \mathrm{~nm}) /$ Bphen $(5 \mathrm{~nm}) / \mathrm{Ag}(100 \mathrm{~nm})$ [see Fig. 1(c)]. Molybdenum-trioxide $\mathrm{MoO}_{3}$ (Merck), $\mathrm{C}_{60}$ (CreaPhys, $2 \times$ sublimed), and Bphen (4,7-diphenyl-1,10-phenanthroline; Fluka) were used as received, whereas ID583 was recrystallized from dimethylformamide. After deposition, the cells were encapsulated (glass-glass package) under inert gas in order to prevent degradation due to the influence of ambient oxygen and humidity. The current-voltage $(J-V)$ characteristics were measured under simulated AM 1.5 illumination. The intensity $\left(100 \mathrm{~mW} / \mathrm{cm}^{2}\right)$ was calibrated using a Si reference cell. For the temperature-dependent measurements, the cells were mounted on a copper block which was temperature controlled by a Peltier element. The cell temperature was measured on the glass substrate directly after each $J-V$ measurement using a contact thermometer. This setup allowed temperatures of the glass substrate between $9{ }^{\circ} \mathrm{C}$ and $48^{\circ} \mathrm{C}$. More details on the device fabrication and the merocyanine synthesis can be found in Ref. 28. 

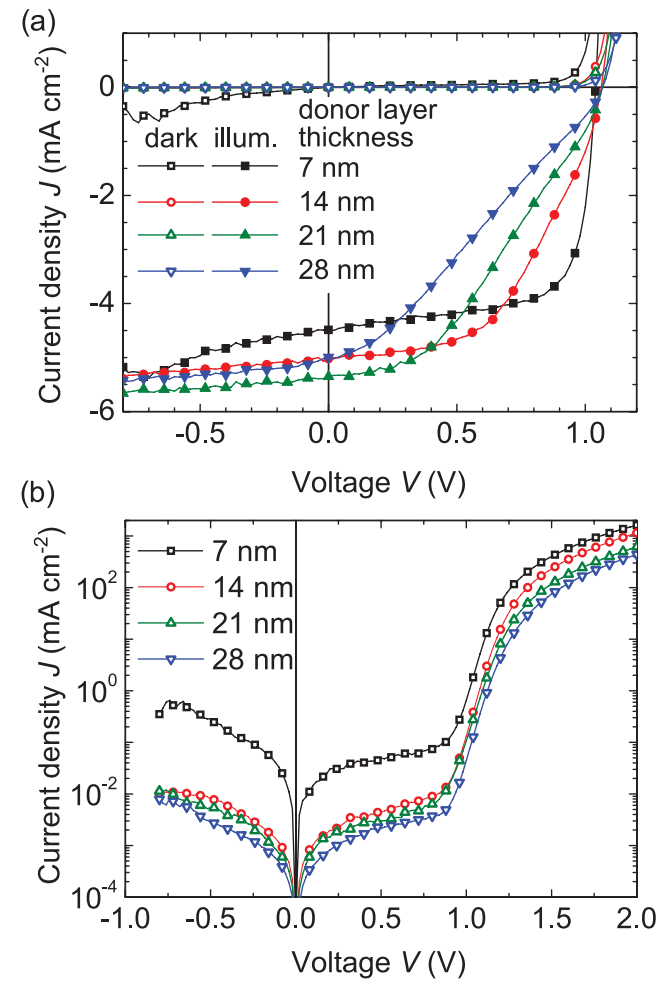

FIG. 2. (Color online) Experimental current-voltage $(J-V)$ characteristics of ID583/ $\mathrm{C}_{60} \mathrm{PHJ}$ cells featuring different thicknesses of the ID583 layer. (a) Data of illuminated and nonilluminated cells on a linear scale. (b) The data of the nonilluminated cells on a semilogarithmic scale.

\section{B. Results}

\section{Influence of the cell thickness}

Figure 2(a) shows the $J-V$ characteristics of $I^{2} 583 / \mathrm{C}_{60}$ PHJ cells for different thicknesses of the donor layer. Table I summarizes the corresponding cell operation parameters. The most prominent feature was the nearly linear current-voltage dependence in the fourth quadrant which drastically reduced the fill factor $(F F)$ of cells with donor thicknesses exceeding $7 \mathrm{~nm}$. The thinnest cells featured a donor layer thickness of only $7 \mathrm{~nm}$ and reached a $F F$ of about $70 \%$. For thicker cells the slope $d J / d V$ of the quasilinear region decreased monotonously with increasing thickness of the donor layer and resulted in fill factors below $30 \%$ for a donor layer thickness of $28 \mathrm{~nm}$. Typically for exciton diffusion limited PHJ cells, the short-circuit current $J_{\mathrm{sc}}$ showed a weak dependence on the device thickness.

In contrast to the strong effect observed under illumination, variations of the donor layer thickness had almost no influence on the $J-V$ characteristics of the nonilluminated cells. Figure 2(b) and Table I show that both the series resistance $R_{\mathrm{S}}$ and the parallel resistance $R_{\mathrm{p}}$ increased with increasing thickness of the donor layer. The shunt current for cells with a donor layer thickness of $7 \mathrm{~nm}$ was about ten times higher than that for the other cells. Although it was visible in the linear scale plot [see Fig. 2(a)], its influence on the $F F$ was negligible. The highest value of the series resistance $R_{\mathrm{s}} \approx 1.8 \Omega \mathrm{cm}^{2}$ was found for cells with a donor layer thickness of $28 \mathrm{~nm}$. At $J_{\mathrm{sc}} \approx 5 \mathrm{~mA} \mathrm{~cm}^{-2}, R_{\mathrm{s}}$ caused a negligible voltage drop of about $9 \mathrm{mV}$. Thus, neither $R_{\mathrm{s}}$ nor $R_{\mathrm{p}}$ constituted a limitation to the $F F$ or the cell performance. The ideality factor $n$ was found to be independent of the donor layer thickness.

\section{Influence of the cell temperature}

Figure 3 shows the experimental $J-V$ characteristics of an ID583/ $\mathrm{C}_{60} \mathrm{PHJ}$ cell with a nominal donor layer thickness of 21 $\mathrm{nm}$, measured at different cell temperatures. Typical for solar cells, the increasing temperature affected both the open-circuit voltage $V_{\text {oc }}$ and the short-circuit current $J_{\text {sc }} \cdot{ }^{36}$ An increase of the cell temperature from $9^{\circ} \mathrm{C}$ to $48^{\circ} \mathrm{C}$ decreased $V_{\text {oc }}$ by about $9 \%$ from 1.11 to $1.01 \mathrm{~V}$ and increased $J_{\text {sc }}$ by about $7 \%$ from $5.81 \mathrm{~mA} \mathrm{~cm}^{-2}$ to $6.21 \mathrm{~mA} \mathrm{~cm}^{-2}$. The obtained temperature coefficient $d V_{\mathrm{oc}} / d T \approx-2.6 \mathrm{mV} \mathrm{K}^{-1}$ for the material system ID583/ $\mathrm{C}_{60}$ was larger than values for other organic $\mathrm{D} / \mathrm{A}$ systems $^{37-39}$ and comparable to the values found in past generations of silicon $p n$-junction solar cells. ${ }^{40}$

In contrast to the pronounced influence of the cell thickness, the cell temperature had nearly no influence on the approximately linear photocurrent-voltage response in the voltage range between 0.3 and $0.9 \mathrm{~V}$.

\section{MODEL}

The experimentally observed thickness dependence of the ID583/ $\mathrm{C}_{60}$ PHJ cells' $J-V$ characteristics occurred only under illumination and was not related to resistive losses. Thus, the observed effect must have been related either to a

TABLE I. Device operation parameters for ID583/C 60 PHJ cells with different thicknesses of the donor layer. The series resistance $R_{\mathrm{s}}$, the parallel resistance $R_{\mathrm{p}}$, and the ideality factor $n$ were determined by fitting a one-diode equivalent circuit model to the $J$ - $V$ characteristics shown in Fig. 2(b).

\begin{tabular}{lccc}
\hline \hline Donor thickness & $7 \mathrm{~nm}$ & $14 \mathrm{~nm}$ & $21 \mathrm{~nm}$ \\
\hline From the dark cells' $J$ - $V$ characteristics & & $28 \mathrm{~nm}$ \\
$R_{\mathrm{s}}\left(\Omega \mathrm{cm}^{2}\right)$ & 0.49 & 0.67 & 1.3 \\
$R_{\mathrm{p}}\left(\Omega \mathrm{cm}^{2}\right)$ & $1.1 \times 10^{4}$ & $1.0 \times 10^{5}$ & $1.5 \times 10^{5}$ \\
$n$ & 1.45 & 1.45 & 1.45 \\
From the illuminated cells' $J$ - $V$ characteristics & & 1.8 \\
$V_{\text {oc }}(\mathrm{mV})$ & 1041 & 1062 & 5.35 \\
$J_{\mathrm{sc}}\left(\mathrm{mA} \mathrm{cm}{ }^{-2}\right)$ & 4.49 & 5.02 & 38.5 \\
$F F(\%)$ & 69.4 & 51.6 & 2.20 \\
$\eta(\%)$ & 3.24 & 2.75 & 1.45 \\
\hline \hline
\end{tabular}




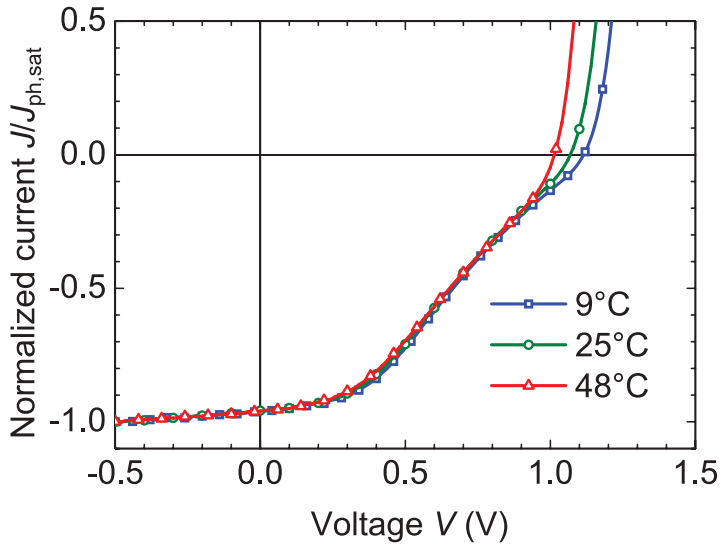

FIG. 3. (Color online) Experimental current-voltage $(J-V)$ characteristics of an ID583/ $\mathrm{C}_{60} \mathrm{PHJ}$ cell with a nominal donor layer thickness of $21 \mathrm{~nm}$, measured at different cell temperatures. The cell temperature had no influence on the approximately linear photocurrent-voltage response in the voltage range between 0.3 and $0.9 \mathrm{~V}$.

field-dependent free charge carrier generation process (geminate recombination) or to field-dependent recombination losses during the collection of the free charge carriers (nongeminate recombination).

In PHJ cells with their well-separated donor and acceptor phases, the internal electric field drives the photogenerated free charge carriers away from the $\mathrm{D} / \mathrm{A}$ interface, leading to a substantial reduction of the nongeminate recombination rate. As a result, the charge carrier collection efficiency in PHJ cells is approximately independent of the applied voltage, and the experimental $J-V$ characteristics of the illuminated cells can be expressed as

$$
J_{\text {illum }}^{\text {sim }}(V)=J_{\text {dark }}^{\text {exp }}(V)-p_{\text {BP }}\left(F_{\text {el }}\right) J_{\text {ph }}^{\text {sat }} .
$$

Here, the experimentally observed dark recombination current and the saturation value of the photocurrent are labeled $J_{\text {dark }}^{\exp }(V)$ and $J_{\mathrm{ph}}^{\mathrm{sat}}$, respectively. The dissociation probability $p_{\mathrm{BP}}\left(F_{\mathrm{el}}\right)$ of the photogenerated bound charge carrier pairs at the D/A interface depends on the electric field $F_{\text {el }}$. Equation (1) is based on the assumption that the nongeminate recombination in PHJ cells does not change with illumination; that is, the nongeminate recombination current density of an illuminated cell is approximately equal to $J_{\text {dark }}^{\exp }(V)$. Drift-diffusion device simulations supported this line of argument. ${ }^{41}$

The internal electric field $F_{\mathrm{el}}$, which is necessary to calculate the bound pair dissociation probability $p_{\mathrm{BP}}$ can be approximated by

$$
F_{\mathrm{el}}=\frac{V-V_{\mathrm{bi}}}{d}
$$

where $d$ and $V_{\mathrm{bi}}$ denote the cell thickness and the built-in voltage, respectively. Equation (2) neglects band bending at the contacts. Thus, it underestimates the value of $F_{\text {el }}$ close to the contacts and slightly overestimates its value in the rest of the cell. However, drift-diffusion simulations verified that Eq. (2) was a valid approximation for the electric field at the $\mathrm{D} / \mathrm{A}$ interface in the critical voltage range $V<0.75 \mathrm{~V}_{\mathrm{bi}}{ }^{41}$

For both types of bound charge carrier pairs, that is, for CT-states and for excitons located at the D/A interface, the field-dependent dissociation probability $p_{\mathrm{BP}}\left(F_{\mathrm{el}}\right)$ is modeled as a competition between dissociation and recombination

$$
p_{\mathrm{BP}}\left(F_{\mathrm{el}}\right)=\frac{k_{\mathrm{diss}}^{\mathrm{BP}}\left(F_{\mathrm{el}}\right)}{k_{\mathrm{diss}}^{\mathrm{BP}}\left(F_{\mathrm{el}}\right)+k_{\mathrm{f}}^{\mathrm{BP}}}=\frac{1}{1+k_{\mathrm{f}}^{\mathrm{BP}} / k_{\mathrm{diss}}^{\mathrm{BP}}\left(F_{\mathrm{el}}\right)},
$$

where $k_{\mathrm{diss}}^{\mathrm{BP}}\left(F_{\mathrm{el}}\right)$ and $k_{\mathrm{f}}^{\mathrm{BP}}$ denote the dissociation and recombination rates, respectively. ${ }^{15}$

In the following, we introduce expressions for the dissociation rates of CT-states $k_{\mathrm{diss}}^{\mathrm{CT}}\left(F_{\mathrm{el}}\right)$ and of excitons $k_{\mathrm{diss}}^{\mathrm{Ex}}\left(F_{\mathrm{el}}\right)$ which are located next to the $\mathrm{D} / \mathrm{A}$ interface.

\section{A. Field-dependent CT-state dissociation model}

The following field-dependent charge-generation model covers the case in which the free charge carrier generation process is limited by the dissociation of the CT-states. For BHJ cells, the field-dependent CT-state dissociation has been modeled using the Onsager-Braun (OB) formalism. ${ }^{15}$ The OB formalism assumes an isotropic active material with $k_{\text {diss }}^{\mathrm{CT}}$ being an even function of the electric field $F_{\mathrm{el}}$. This assumption is not valid in PHJ solar cells with their geometrically well-defined $\mathrm{D} / \mathrm{A}$ interface structure, where electrons and holes have to escape from the bound pairs into their respective material phases over two half-spheres.

Figure 4 shows a schematic of the bound pair dissociation process in PHJ cells. The total bound pair dissociation rate at a planar D/A interface has to be calculated as an integral over all possible directions of escape. For the CT-states it can be written as

$$
\begin{aligned}
k_{\text {diss }}^{\mathrm{CT}}\left(E_{\text {field }}>E_{\mathrm{b}}^{\mathrm{CT}}\right)= & A_{\text {diss }}^{\mathrm{CT}}, \\
k_{\text {diss }}^{\mathrm{CT}}\left(E_{\text {field }} \leqslant E_{\mathrm{b}}^{\mathrm{CT}}\right)= & \frac{A_{\text {diss }}^{\mathrm{CT}}}{2 \pi} \int_{0}^{2 \pi} d \varphi \int_{0}^{\pi / 2} \sin (\theta) \\
& \times \exp \left(\frac{-E_{\mathrm{b}}^{\mathrm{CT}}+E_{\text {field }}}{k_{\mathrm{B}} T}\right) d \theta,
\end{aligned}
$$

where $A_{\text {diss }}^{\mathrm{CT}}$ is the attempt to escape frequency, while $\theta$ denotes the angle between the electric field $F_{\text {el }}$ and the direction of escape. ${ }^{7}$ The CT-state binding energy

$$
E_{\mathrm{b}}^{\mathrm{CT}}=E_{\mathrm{e} / \mathrm{h}}-E_{\mathrm{CT}}
$$

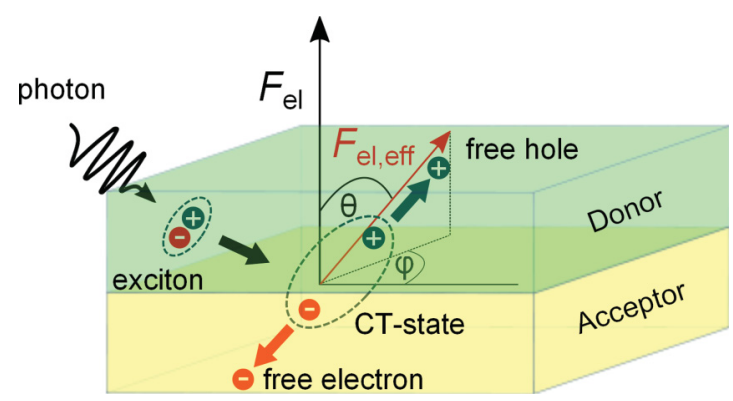

FIG. 4. (Color online) Schematic of the charge-generation process in organic PHJ solar cells. The photogenerated excitons diffuse toward the D/A interface where they can form CT-states. Electrons and holes can escape over two half spheres into their respective material phases. For each direction of escape, the bound pairs' dissociation is supported by an effective field $F_{\text {el,eff }}=F_{\text {el }} \cos (\theta)$. 
is defined as the difference between the energy of the CT-state $E_{\mathrm{CT}}$ and the energy of a free electron-hole pair $E_{\mathrm{e} / \mathrm{h}}$. The electric field vector $\vec{F}_{\text {el }}$ is assumed to be oriented perpendicular to the $\mathrm{D} / \mathrm{A}$ interface. It exerts a force on the bound charges which is proportional to the field component that is oriented parallel with the direction of escape. Hence, the electric field $F_{\text {el }}$ lowers $E_{\mathrm{b}}^{\mathrm{CT}}$ by

$$
E_{\text {field }}=-q F_{\mathrm{el}} \cos (\theta) r_{\mathrm{s}},
$$

where $q$ represents the elementary charge. The separation distance $r_{\mathrm{s}}$ can be viewed as the additional distance by which the electron and hole in a CT-state have to move apart in order to be considered free. This means that the CT-state dissociates as soon as its spatial extent exceeds a critical value $r_{\text {crit }}=r_{0}+r_{\mathrm{s}}$, where $r_{0}$ is the initial extent of the CT-state. Due to the nonsymmetric shape of the donor molecules [see Fig. 1(a)], it is likely that the separation distance is an anisotropic quantity. Thus, the parameter $r_{\mathrm{s}}$ represents a mean or effective value of the separation distance and controls the influence of the electric field on the CT-state dissociation process. Evaluating the integral in Eq. (4) yields

$$
k_{\mathrm{diss}}^{\mathrm{CT}}\left(E_{\mathrm{b}}^{\mathrm{CT}}, F_{\mathrm{el}}, T\right)=k_{\mathrm{diss}, 0}^{\mathrm{CT}}\left(E_{\mathrm{b}}^{\mathrm{CT}}, T\right) B_{\mathrm{CT}}\left(F_{\mathrm{el}}, T\right),
$$

with

$$
\begin{gathered}
k_{\mathrm{diss}, 0}^{\mathrm{CT}}\left(E_{\mathrm{b}}^{\mathrm{CT}}, T\right)=A_{\mathrm{diss}}^{\mathrm{CT}} \exp \left(-\frac{E_{\mathrm{b}}^{\mathrm{CT}}}{k_{\mathrm{B}} T}\right), \\
B_{\mathrm{CT}}\left(F_{\mathrm{el}}, T\right)=\frac{\left[1-\exp \left(-F_{\mathrm{el}} q r_{\mathrm{s}} / k_{\mathrm{B}} T\right)\right] k_{\mathrm{B}} T}{F_{\mathrm{el}} q r_{\mathrm{s}}},
\end{gathered}
$$

and

$$
\lim _{F_{\mathrm{el}} \rightarrow 0} B_{\mathrm{CT}}\left(F_{\mathrm{el}}, T\right)=1 .
$$

The field-dependent term $B_{\mathrm{CT}}\left(F_{\mathrm{el}}, T\right)$ is a monotone and positive function of the electric field $F_{\text {el }}$. Under normal cell operation conditions, that is, $V<V_{\mathrm{bi}}$ and $F_{\mathrm{el}}<0$ [see Eq. (2)], $B_{\mathrm{CT}}\left(F_{\text {el }}, T\right)$ is greater than unity and increases for decreasing values of the applied voltage $V$.

According to Eq. (3), the resulting CT-state dissociation probability $p_{\mathrm{CT}}$ can be expressed as

$$
p_{\mathrm{CT}}\left(F_{\mathrm{el}}, T\right)=\frac{1}{1+\frac{k_{\mathrm{f}}^{\mathrm{CT}}}{k_{\mathrm{diss}, 0}^{\mathrm{CT}}(T)} \frac{1}{B_{\mathrm{CT}}\left(F_{\mathrm{el}}, T\right)}} .
$$

Alternatively, after applying Eq. (8), it can also be written as

$$
p_{\mathrm{CT}}\left(F_{\mathrm{el}}, T\right)=\frac{1}{1+\frac{k_{\mathrm{f}}^{\mathrm{CT}}}{A_{\mathrm{diss}}^{\mathrm{CT}}} \frac{\exp \left[E_{\mathrm{b}}^{\mathrm{CT}} /\left(k_{\mathrm{B}} T\right)\right]}{B_{\mathrm{CT}}\left(F_{\mathrm{el}}, T\right)}} .
$$

Equation (11) is the more practical form if $J-V$ data are available for only one value of the cell temperature. In this case, the effect of the CT-state binding energy $E_{\mathrm{b}}^{\mathrm{CT}}$ cannot be distinguished from that of the rate constants $k_{\mathrm{f}}^{\mathrm{CT}}$ and $A_{\text {diss }}^{\mathrm{CT}}$. Hence, for fitting purposes, all three can be taken together as a single fit parameter: the loss ratio $k_{\mathrm{f}}^{\mathrm{CT}} / k_{\mathrm{diss}, 0}^{\mathrm{CT}}$, which is the ratio of the CT-state's recombination rate $k_{\mathrm{f}}^{\mathrm{CT}}$ and its

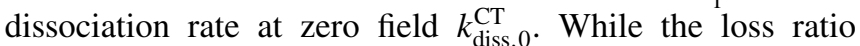
$k_{\mathrm{f}}^{\mathrm{CT}} / k_{\mathrm{diss}, 0}^{\mathrm{CT}}$ determines the CT-state dissociation probability $p_{\mathrm{CT}}$ at zero field, the separation distance $r_{\mathrm{s}}$ controls how fast $p_{\text {CT }}$ changes with the electric field. The two parameters are linearly independent.
If temperature-dependent data are available, Eq. (12) is more appropriate because it allows one to split the loss ratio into two fit parameters: the loss ratio's temperatureindependent prefactor $k_{\mathrm{f}}^{\mathrm{CT}} / A_{\text {diss }}^{\mathrm{CT}}$ and the CT-state binding energy $E_{\mathrm{b}}^{\mathrm{CT}}$. Except for the field-dependent term $B_{\mathrm{CT}}\left(F_{\mathrm{el}}, T\right)$, the above-mentioned model has the same functional form as the OB formalism. ${ }^{15}$

\section{B. Field-dependent tunnel model for the dissociation of excitons}

In this section, we present a field-dependent chargegeneration model covering the case in which the free charge carrier generation process is limited by the initial dissociation of the exciton rather than by the dissociation of the CTstate. Because the exciton dissociation is an energy-releasing process, an energy barrier hindering the dissociation can be tunneled. In contrast to the Miller-Abrahams hopping, ${ }^{42}$ which is a combination of a field-independent tunneling term and a field-dependent Boltzmann factor and has been utilized to model the CT-state dissociation at the D/A interface, ${ }^{19,43,44}$ we propose a field-dependent tunnel process which takes into account the polarizability of the excited donor molecules.

Figure 5 shows a schematic of the field-dependent tunnel process. Assuming that the optically excited molecules are polarized by the electric field $F_{\text {el }},{ }^{45}$ which is given by Eq. (2), their dipole moments $p$ increase by $\Delta p=-\alpha F_{\text {el }}$, where $\alpha$ denotes an effective value of the excited state's polarizability in the direction of the applied electric field. As a result, the spatial

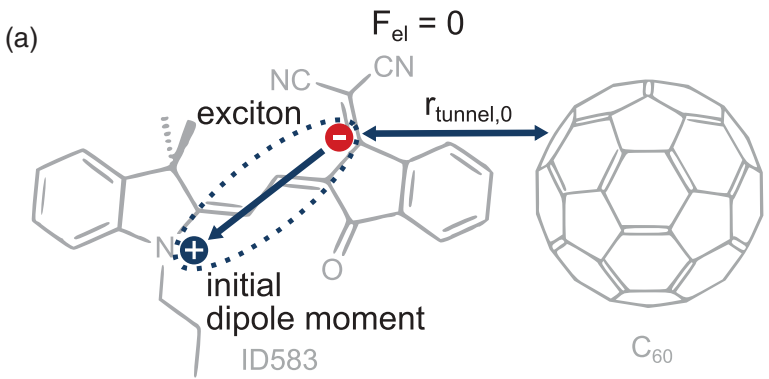

(b)

$$
\text { donor molecule }
$$

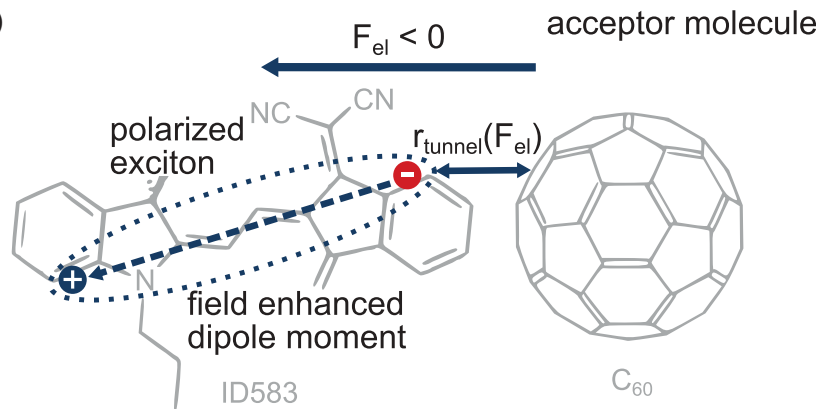

FIG. 5. (Color online) Schematic of the field-dependent tunnel process at the ID583/ $\mathrm{C}_{60}$ heterojunction. Due to the ID583 molecules' dipole moment (Ref. 28) the donor exciton's negative and positive charges are located close to the two nitrile groups and the propyl group, respectively. (a) Without an electric field $\left(F_{\mathrm{el}}=0\right)$, the exciton dissociation requires the electron to tunnel over a distance $r_{\text {tunnel, },}$. (b) An external field $F_{\text {el }}<0$ polarizes the excited state and thereby reduces $r_{\text {tunnel }}$. 
extent of the excitons increases by $\Delta r=\Delta p / q$. In a first-order approximation, electron and hole are each shifted by the same distance but in opposite directions and the tunnel distance $r_{\text {tunnel }}$ is reduced by $\Delta r / 2$. The resulting field-dependent tunnel distance reads

$$
r_{\text {tunnel }}\left(F_{\mathrm{el}}\right)=r_{\text {tunnel, } 0}-\frac{-\alpha F_{\mathrm{el}}}{2 q},
$$

with the constraint $r_{\text {tunnel }}\left(F_{\text {el }}\right) \geqslant 0$; that is, a field dependence occurs only as long as

$$
\frac{-\alpha F_{\mathrm{el}}}{2 q} \leqslant r_{\text {tunnel, } 0}
$$

Although $r_{\text {tunnel }}$ does not explicitly depend on the exciton binding energy, the latter is implicitly included in the exciton size and thus in both the polarizability $\alpha^{46,47}$ and the zero field tunnel distance $r_{\text {tunnel, } 0}$. In the model, a more detailed expression for $r_{\text {tunnel }}$ is not useful because it would increase the number of unknown parameters and result in an overparameterized fit.

The tunnel rate $A \exp \left(-2 \gamma r_{\text {tunnel }}\right)$ depends on the attempt to escape frequency $A$ and the inverse localization radius $\gamma \cdot{ }^{48}$ An integration over all possible directions of escape, as described in Fig. 4 and Eq. (4), yields the exciton dissociation rate

$$
k_{\mathrm{diss}}^{\mathrm{Ex}}=k_{\mathrm{diss}, 0}^{\mathrm{Ex}} \int_{0}^{\pi / 2} \sin (\theta) \exp \left(-\frac{\gamma \alpha F_{\mathrm{el}} \cos (\theta)}{q}\right) d \theta,
$$

with $k_{\mathrm{diss}, 0}^{\mathrm{Ex}}=A \exp \left(-2 \gamma r_{\text {tunnel, } 0}\right)$. Equation (15) has the same functional form as Eq. (4) but does not depend on the device temperature. A comparison of coefficients yields

$$
k_{\mathrm{diss}, 0}^{\mathrm{Ex}}=k_{\mathrm{diss}, 0}^{\mathrm{CT}}
$$

and

$$
\gamma \alpha=\frac{q^{2} r_{\mathrm{s}}}{k_{\mathrm{B}} T}
$$

Thus, for any fixed value of the device temperature, the exciton dissociation model and the CT-state dissociation model can be parameterized with the equivalent sets of parameters. As a result, the two introduced field-dependent charge-generation models can be distinguished only by their temperature dependences.

Analogous to Eq. (11), the field-dependent exciton dissociation efficiency can be expressed as

$$
p_{\mathrm{Ex}}\left(F_{\mathrm{el}}\right)=\frac{1}{1+\frac{k_{\mathrm{f}}^{\mathrm{Ex}}}{k_{\mathrm{diss}, 0}^{\mathrm{Ex}}} \frac{1}{B_{\mathrm{Ex}}\left(F_{\mathrm{el}}\right)}},
$$

with

$$
B_{\mathrm{Ex}}\left(F_{\mathrm{el}}\right)=\frac{\left[1-\exp \left(-F_{\mathrm{el}} \gamma \alpha / q\right)\right] q}{F_{\mathrm{el}} \gamma \alpha} .
$$

\section{DISCUSSION}

\section{A. Influence of the cell thickness}

In order to verify the models' compatibility with the experimental data, we fitted them to the thickness-dependent $J-V$ characteristics which are shown in Fig. 2(a). For this purpose, we expressed the current of the illuminated cells

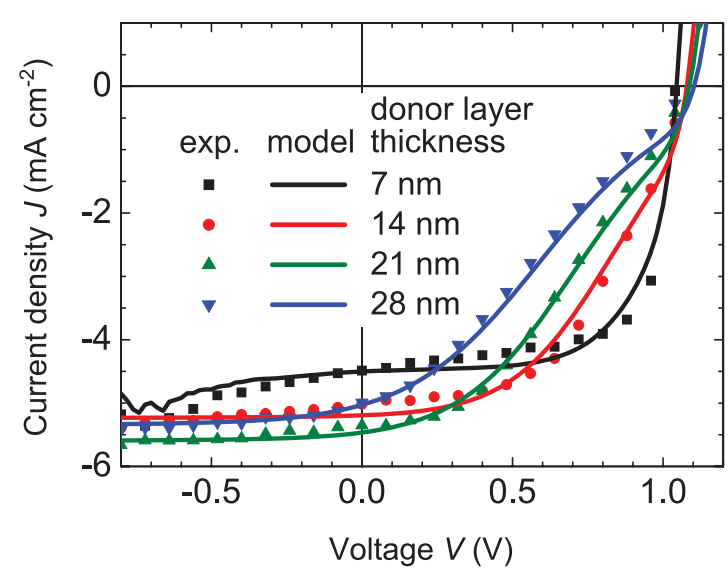

FIG. 6. (Color online) Comparison between experimental (symbols) and modeled (solid lines) current-voltage $(J-V)$ characteristics of ID583/ $\mathrm{C}_{60}$ PHJ cells featuring different thicknesses of the ID583 layer. The calculations were performed using the two field-dependent charge carrier generation models introduced in Secs. III A and III B. Table II summarizes the model parameters.

according to Eqs. (1) and (2), where the bound pair dissociation probability was modeled according to Eqs. (11) and (18), which are equivalent in the present case of a fixed device temperature (see Sec. III B).

The active layer thickness $d=d_{\text {donor }}+d_{\text {acceptor }}$ was chosen within the limits of the experimental uncertainty $( \pm 10 \%)$ and the thin $\mathrm{MoO}_{3}$ and Bphen layers were treated as parts of the anode and the cathode, respectively.

Figure 6 shows the excellent agreement between the two field-dependent charge-generation models and the experimental data. The $J-V$ characteristics of the four cells were modeled using a common set of parameters which is summarized in Table II. For the cells featuring a donor layer thickness of $7 \mathrm{~nm}$, the electric field $F_{\text {el }}$ was increased by $15 \%$ compared to the value obtained by Eq. (2). Without this adjustment the models strongly underestimated the $F F$ of the thinnest devices. This enhanced value of $F_{\mathrm{el}}$ could be attributed to band bending at the anode. The low injection barrier at the anode of the ID583/ $\mathrm{C}_{60}$ devices caused a charge accumulation and thus a band bending. ${ }^{27,49}$ In the cells with a $7 \mathrm{~nm}$ donor layer, the $\mathrm{D} / \mathrm{A}$ interface, where the charge dissociation occurred, was located very close to the anode. As a result, the increased field in this region enhanced the charge dissociation process.

Two free parameters were used in the fitting process. The first was the loss ratio $k_{\mathrm{f}} / k_{\text {diss, } 0}$, which had identical values in the exciton dissociation model and in the CT-state dissociation model, that is, $k_{\mathrm{f}}^{\mathrm{CT}} / k_{\mathrm{diss}, 0}^{\mathrm{CT}}=k_{\mathrm{f}}^{\mathrm{Ex}} / k_{\mathrm{diss}, 0}^{\mathrm{Ex}}$. For the models' loss ratios, the fit yielded a value of 31 . This value implied a bound pair dissociation probability at zero internal field of $1 / 32 \approx$ $3.1 \%$ [see Eq. (3)]. Under operating conditions, the internal electric field $F_{\mathrm{el}}$ supported the charge carrier dissociation processes and much higher dissociation probabilities were obtained. Because $F_{\text {el }}$ decreased with increasing cell thickness [see Eq. (2)], the field-assisted charge dissociation probability exhibited a strong dependence on the device thickness. For example, at $V=0.5 \mathrm{~V}$ the model predicted a bound pair dissociation probability of $89 \%$ for a cell with a nominal donor thickness $d_{\text {donor }}=14 \mathrm{~nm}$, while $d_{\text {donor }}=21 \mathrm{~nm}$ and 
TABLE II. Model parameters used for the simulation of the thickness-dependent current-voltage $(J-V)$ characteristics shown in Fig. 6.

\begin{tabular}{|c|c|c|c|}
\hline Parameter & Symbol & Value & Origin \\
\hline Built-in voltage & $V_{\mathrm{bi}}$ & $1.54 \mathrm{~V}$ & Exp. $^{\mathrm{a}}$ \\
\hline Acceptor thickness & $d_{\text {acceptor }}$ & $31.5 \mathrm{~nm}$ & Exp. $^{\mathrm{b}}$ \\
\hline Donor thickness & $d_{\text {donor }}$ & 7.7 to $30.8 \mathrm{~nm}$ & Exp. $^{c}$ \\
\hline Saturation value of the photocurrent & $J_{\mathrm{ph}, \mathrm{sat}}$ & 4.5 to $5.6 \mathrm{~mA} \mathrm{~cm}^{-2}$ & Exp. $^{\mathrm{d}}$ \\
\hline Temperature & $T$ & $298 \mathrm{~K}$ & Exp. $^{\mathrm{e}}$ \\
\hline \multicolumn{4}{|c|}{ Parameters specific to the CT-state dissociation model } \\
\hline CT-state loss ratio & $k_{\mathrm{f}}^{\mathrm{CT}} / k_{\mathrm{diss}, 0}^{\mathrm{CT}}$ & 31 & Fit \\
\hline Separation distance & $r_{\mathrm{s}}$ & $8.7 \mathrm{~nm}$ & Fit \\
\hline \multicolumn{4}{|c|}{ Parameters specific to the exciton dissociation model } \\
\hline Exciton loss ratio & $k_{\mathrm{f}}^{\mathrm{Ex}} / k_{\mathrm{diss}, 0}^{\mathrm{Ex}}$ & 31 & Eq. (16) \\
\hline Tunnel coefficient & $\gamma \alpha$ & $5.4 \times 10^{-26} \mathrm{C} \mathrm{mV}^{-1}$ & Eq. (17) \\
\hline
\end{tabular}

${ }^{a}$ Calculated from the energy difference between the cathode work function and the donor HOMO (assuming Fermi level pinning at the anode).

${ }^{\mathrm{b}} 90 \%$ of the nominal value (the experimental uncertainty of the layer thicknesses is $10 \%$ ).

${ }^{\mathrm{c}} 110 \%$ of the nominal value (the experimental uncertainty of the layer thicknesses is $10 \%$ ).

${ }^{\mathrm{d}}$ Experimental current at $V=-0.5 \mathrm{~V}$ for donor layer thicknesses $d_{\text {donor }}>7 \mathrm{~nm}$. For $d_{\text {donor }}=7 \mathrm{~nm}$ the value at $V=0 \mathrm{~V}$ is taken in order to avoid an influence of the low parallel resistance observed in these cells.

${ }^{\mathrm{e}}$ Temperature of the glass substrate.

$d_{\text {donor }}=28 \mathrm{~nm}$ resulted in dissociation probabilities of $76 \%$ and $62 \%$, respectively.

In both models, the respective second fit parameters controlled the influence of the electric field on the chargegeneration process. For the CT-state dissociation model, the value obtained for the separation distance $r_{\mathrm{s}}=8.7 \mathrm{~nm}$ was similar to the values predicted by Barker et al. ${ }^{7}$ They assumed that the CT-state dissociates as soon as its spatial extent reached the top of the energy barrier given by the superposition of the charges' mutual Coulomb potential and internal electric field, that is, $r_{\mathrm{s}}\left(F_{\mathrm{el}}\right)=\sqrt{-q /\left(4 \pi \epsilon_{\mathrm{r}} \epsilon_{0} F_{\mathrm{el}}\right)}$. For cells with a nominal donor thickness of $21 \mathrm{~nm}$, this simple Coulomb model resulted in $3.9 \mathrm{~nm} \leqslant r_{\mathrm{s}} \leqslant 6.5 \mathrm{~nm}$ for $0 \mathrm{~V} \leqslant V \leqslant 1 \mathrm{~V}$, assuming a relative permittivity $\epsilon_{\mathrm{r}}=3.4$.

For the exciton dissociation model, the second fit parameter, that is, the tunnel coefficient $\gamma \alpha \approx 5.4 \times 10^{-26} \mathrm{C} \mathrm{mV}^{-1}$ (see Table II), was the product of the excited state's polarizability $\alpha$ and the inverse localization radius $\gamma$. In order to disentangle the two parameters and to allow for a comparison with values reported in the literature, we estimated an upper limit for $\alpha$ using the inequality Eq. (14). The required value of the zero field tunnel distance $r_{\text {tunnel }, 0}=0.6 \mathrm{~nm}$ was estimated from the exciton's position on the donor molecule and the dimensions of the molecule [see Fig. 5(a)]. The electric field $F_{\mathrm{el}}=-2.8 \times 10^{7} \mathrm{~V} \mathrm{~m}^{-1}$ at which the photogeneration saturated was calculated for $V=0 \mathrm{~V}$ and a donor layer thickness of $21 \mathrm{~nm}$ [see Fig. 6 and Eq. (2)].

The obtained upper limit for the polarizability $\alpha \leqslant 7 \times$ $10^{-36} \mathrm{C} \mathrm{m}^{2} \mathrm{~V}^{-1}$ was about one to two orders of magnitude larger than experimental values of the excited state's polarizability of pentacene, symmetrical porphyrin oligomers, and vanadyl naphthalocyanine. ${ }^{47,50,51}$ This deviation did not contradict the validity of our fit because the obtained estimate only represented an upper limit for the polarizability $\alpha$ in our model. Additionally, the above-mentioned experimental values were not obtained from solar-cell-like structures but from sub-monolayer samples, ${ }^{47}$ from solutions in toluene, ${ }^{51}$ and from the gas phase..$^{50}$ Taking into account that not only the molecular structure ${ }^{52}$ but also the polarity of the molecules' surroundings influences the polarizability, ${ }^{53}$ the agreement between the experimental values and the estimate based on our fit parameters was rather good.

An estimate for the inverse localization radius $\gamma$ was calculated using the above-mentioned upper limit for $\alpha$ and the fit value for the tunnel coefficient $\gamma \alpha=5.4 \times 10^{-26} \mathrm{C} \mathrm{mV}^{-1}$ (see Table II). The obtained lower limit for the inverse localization radius $\gamma \geqslant 7.7 \times 10^{9} \mathrm{~m}^{-1}$ was plausible and agreed with values used for modeling in the literature. ${ }^{19}$ Note that the above-mentioned estimates for $r_{\text {tunnel, }, 0}, \alpha$, and $\gamma$ were used to check the plausibility of the obtained fit parameter values. They were not used in the fitting process.

In summary, both charge-generation models were consistent with the voltage and thickness-dependent current-voltage $(J-V)$ characteristics of the ID583/ $\mathrm{C}_{60} \mathrm{PHJ}$ solar cells. Also, the OB model, ${ }^{15}$ which describes the field-dependent CT-state dissociation in BHJ cells, yielded a fit of similar quality as the one displayed in Fig. $6 .^{41}$

\section{B. Influence of the cell temperature}

The experimental data on the variation of the cell thickness proved to be insufficient to determine whether the chargegeneration process in the ID583/ $\mathrm{C}_{60} \mathrm{PHJ}$ cells was limited by the CT-state dissociation or by the initial dissociation of the excitons. In this section, we check the models' agreement with $J-V$ characteristics measured at different cell temperatures.

While the proposed exciton dissociation model does not feature any additional free parameter that could be fitted to the temperature dependence, the loss ratio $k_{\mathrm{f}}^{\mathrm{CT}} / k_{\mathrm{diss}, 0}^{\mathrm{CT}}$ of the CT-state dissociation model depends on the cell temperature [see Eq. (8)]. In order to guarantee compatibility with the thickness-dependent data shown in Fig. 6 and the parameters in Table II, we substituted $k_{\mathrm{f}}^{\mathrm{CT}} / A_{\mathrm{diss}}^{\mathrm{CT}}$ in Eq. (12) with

$$
\frac{k_{\mathrm{f}}^{\mathrm{CT}}}{A_{\mathrm{diss}}^{\mathrm{CT}}}=31 \exp \left(-\frac{E_{\mathrm{b}, \mathrm{CT}}}{k_{\mathrm{B}} 298 \mathrm{~K}}\right),
$$


(a) CT-state dissociation model
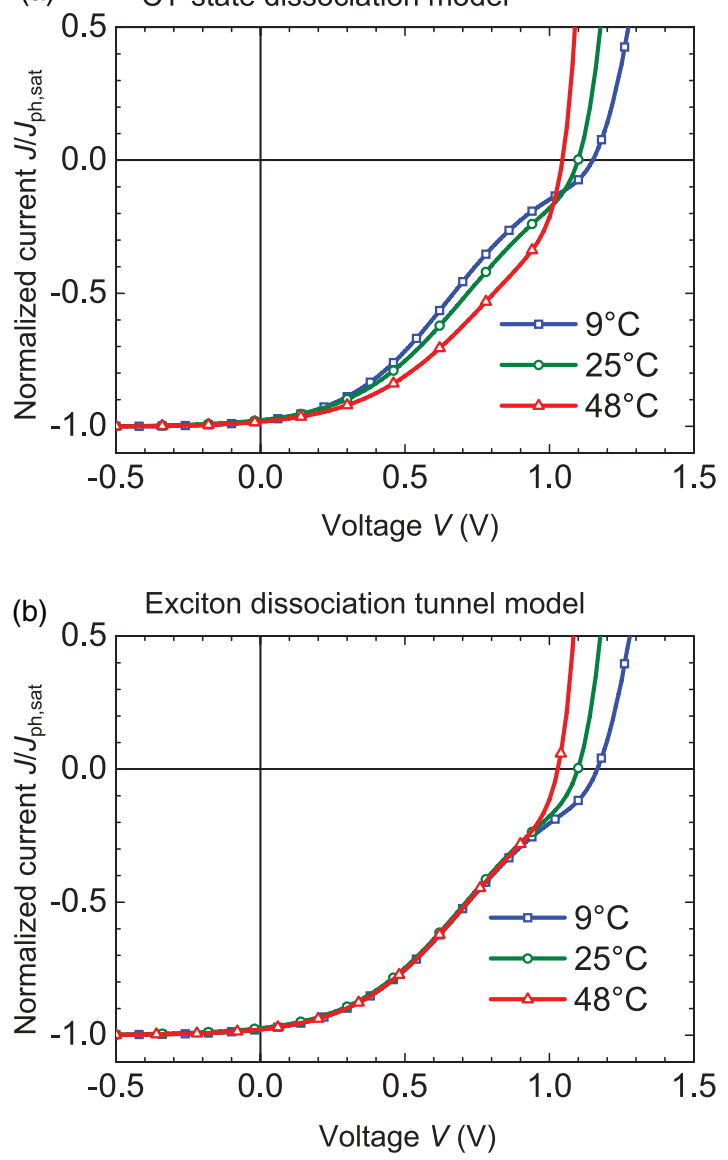

FIG. 7. (Color online) Modeled temperature-dependent currentvoltage $(J-V)$ characteristics of an ID583-based PHJ cell with a nominal donor layer thickness of $21 \mathrm{~nm}$. (a) Calculation according to the field-dependent CT-state dissociation model presented in Sec. III A. Although the CT-state binding energy $E_{\mathrm{b}, \mathrm{CT}}=245 \mathrm{meV}$ was chosen such that it yielded the best agreement with the experimental data (see the text for details), this model strongly overestimated the influence of the cell temperature (compare Fig. 3). The temperature-independent prefactor $k_{\mathrm{f}} / A_{\text {diss }}=2.2 \times 10^{-3}$ was calculated according to Eq. (20). (b) Calculation according to the field-dependent exciton dissociation model (see Sec. III B), which reproduced the experimentally observed temperature dependence shown in Fig. 3. In this model, the only temperature dependence originated from the experimentally obtained temperature-dependent dark recombination current $J_{\text {dark }}^{\exp }$ [see Eq. (1)]. Table II summarizes all utilized model parameters.

which was obtained by multiplying Eq. (8) by $k_{\mathrm{f}}^{\mathrm{CT}}$ and inserting the values for the loss ratio and the temperature from Table II. That way, the exponential terms in Eq. (12) canceled at $T=298 \mathrm{~K}$ and the loss ratio $k_{\mathrm{f}}^{\mathrm{CT}} / k_{\text {diss }, 0}^{\mathrm{CT}}=31$, which had been obtained from the thickness-dependent measurements, was recovered.

Figure 7(a) displays $J$ - $V$ characteristics calculated according to the field-dependent CT-state dissociation model from Sec. III A. In contrast to the experimental data shown in Fig. 3, the CT-state dissociation model predicted a pronounced temperature dependence of the charge carrier generation process. The model's temperature dependence was minimized by setting the CT-state binding energy $E_{\mathrm{b}, \mathrm{CT}}=245 \mathrm{meV}$ to the lowest value that was compatible with the experimentally observed field dependence at voltages $V>0 \mathrm{~V}$. According to Eq. (4), the CT-state dissociation exhibited a field dependence only as long as the charges had to overcome an energy barrier, that is, only as long as $E_{\text {field }} \leqslant E_{\mathrm{b}, \mathrm{CT}}$. For this reason, $E_{\mathrm{b}, \mathrm{CT}}$ was chosen such that it equaled the absolute value of $E_{\text {field }}$ at an applied voltage $V=0 \mathrm{~V}$. That way, it was guaranteed that $E_{\text {field }} \leqslant E_{\mathrm{b}, \mathrm{CT}}$ for voltages $V>0 \mathrm{~V}$.

The loss ratio of the OB model featured a similar exponential temperature dependence as in the CT-state dissociation model presented here. For this reason, the OB model was also incompatible with the experimental data. ${ }^{41}$

In contrast to the field-dependent CT-state dissociation models, the field-dependent exciton dissociation tunnel model was able to reproduce the experimentally observed temperature dependence without the need for an additional fit parameter [see Fig. 7(b)]. Thus, the proposed exciton dissociation model simultaneously explained the influence of the applied voltage, the device thickness, and the temperature on the $J$ - $V$ characteristics of the ID583/ $\mathrm{C}_{60} \mathrm{PHJ}$ cells.

\section{Alternative explanations for a weak temperature dependence}

The above-mentioned results indicated that neither the CT-state dissociation model for PHJ solar cells nor the OB model could account for the experimentally observed combination of a high field dependence and a practically nonexistent temperature dependence. We argued that both models overestimated the temperature dependence because they treated the CT-state dissociation as an escape process over an energy barrier using a Boltzmann term. In this section, we discuss other explanations for a weak temperature dependence that have been published in the literature.

In most cases, the weak temperature dependence of the CT-state dissociation process was attributed to additional driving forces for the charge separation such as dipoles ${ }^{54}$ and multipoles $^{55}$ at the donor acceptor interface, entropy effects, ${ }^{3}$ relaxation processes in energetically disordered densities of states, ${ }^{4,48,56}$ charge delocalization on polymer chains, ${ }^{19,44}$ nonthermalized (hot) CT-states, ${ }^{57-62}$ and high local values of the mobility. ${ }^{17}$ Such additional driving forces increase the CT-state dissociation probability $p_{\mathrm{CT}}$, and if a cell is not limited by the CT-state dissociation, the temperature induced changes of the dissociation efficiency hardly influence the current-voltage $(J-V)$ characteristics. Thus, additional driving forces can only account for a simultaneous occurrence of a weak field and temperature dependence. This combination is typical for state-of-the-art organic BHJ solar cells which are not limited by the geminate recombination of CT-states, ${ }^{63,64}$ but mainly by nongeminate recombination of free charge carriers. $^{23-26}$

Another proposed mechanism which could explain a weak temperature dependence was a dissociation process involving disorder-assisted tunneling jumps. ${ }^{65}$ In contrast to this theory, a weak temperature dependence was found for both strongly and weakly disordered materials. ${ }^{66,67}$ Later, it was proposed that the excess energy of the optical excitation may generate a heat bath on the donor molecules which could be characterized by an effective temperature $T_{\text {eff }}>T_{\text {cell }}{ }^{68-70}$ By replacing the cell temperature $T_{\text {cell }}$ by $T_{\text {eff }}$, the authors made the Boltzmann term 
independent of $T_{\text {cell }}$. However, sufficiently large thermalization times of the donor molecules require large uninterrupted $\pi$ systems, that is, long conjugated polymer chains. ${ }^{69}$ Thus, the relatively short donor molecules which were utilized in this paper most likely thermalized before they could transfer their energy to the bound electron-hole pairs.

\section{Influence of the dye orientation on the exciton dissociation efficiency}

We recently published that the distinct dependence of the $J-V$ characteristics on the applied voltage and device thickness abruptly disappeared after annealing the ID583/ $\mathrm{C}_{60}$ PHJ cells at a temperature above the donor's glass transition temperature. ${ }^{28}$ The annealing was found to change the donor molecules' orientation, which decreased the CT-state binding energy and made the annealing effect compatible with the CT-state dissociation model presented in Sec. III A. ${ }^{28}$ Here, we demonstrate that also the proposed exciton dissociation tunnel model can account for the observed relation between dye orientation and charge-generation efficiency.

The ID583 molecules were found to exhibit a permanent ground-state dipole moment of $7.1 \mathrm{D}$ along the molecules long axis and $5.5 \mathrm{D}$ along the short axis. ${ }^{28}$ Thus, the total dipole moment of a ID583 molecule had a value of 9.0 D and was oriented at an angle of $38^{\circ}$ relative to the long axis of the molecule, pointing from the nitrile groups attached to the indane toward the propyl group attached to the indoline [see Fig. 5(a)]. During the annealing process, the donor molecules, initially oriented with their long axis perpendicular to the substrate surface, rotated by $45^{\circ} .28$ Thus, the dipole moment's orientation changed from its initial value of $38^{\circ}$ to $-7^{\circ}$ relative to the substrate's surface normal. With the cell's internal electric field now oriented in almost the same direction as the intrinsic dipole moment, the high effective polarizability $\alpha$ along the direction of the intrinsic dipole moment and the reduced initial tunnel distance $r_{\text {tunnel, } 0}$ are likely explanations for the increase in dissociation probability which was observed upon annealing.

\section{CONCLUSION}

We have demonstrated that the PHJ cell configuration features a virtually field-independent charge carrier collection efficiency and constitutes a convenient model system to study the field dependence of the charge carrier generation process in organic heterojunction solar cells. This model system was applied to test the consistency of current CT-state dissociation models with experimental data of PHJ cells made of a merocyanine dye (ID583) and the fullerene $\mathrm{C}_{60}$ as donor and acceptor, respectively. These devices exhibited a distinct field-dependent charge-generation process which could be observed during normal, steady-state solar-cell operation. Although the CT-state dissociation models quantitatively reproduced the photocurrent's dependence on the applied voltage and the device thickness, they failed to account for the virtually negligible temperature dependence of the field-dependent charge-generation process. The reason for the observed discrepancy was a common feature of the models: an Arrhenius-like temperature dependence, distinctive of all processes involving a thermally activated jump over an energy barrier. As a possible solution to this problem, we introduced an exciton dissociation model based on a field-dependent tunnel process and demonstrated its consistency with the experimental observations. Future work needs to apply the proposed combination of device thickness and temperaturedependent measurements on PHJ cells to identify other D/A combinations that exhibit a field-dependent charge-generation process.

\section{ACKNOWLEDGMENTS}

We would like to thank Robert Gustafson, Stefan Schäfer, and Minh Nguyen for carefully reading the manuscript and Andreas Fuchs for helpful discussions on the calculations of energy levels. We also thank the BMBF for the financial support in the framework of the OPEG Project (13N9715). T.K. acknowledges support by an Imperial College Junior Research Fellowship.

\footnotetext{
*andreas.petersen@de.bosch.com

${ }^{1}$ M. A. Green, K. Emery, Y. Hishikawa, W. Warta, and E. D. Dunlop,

Prog. Photovoltaics 20, 12 (2012).

${ }^{2}$ C. Deibel and V. Dyakonov, Rep. Prog. Phys. 73, 096401 (2010).

${ }^{3}$ T. M. Clarke and J. R. Durrant, Chem. Rev. 110, 6736 (2010).

${ }^{4}$ C. Deibel, T. Strobel, and V. Dyakonov, Adv. Mater. 22, 4097 (2010).

${ }^{5}$ C. W. Tang, Appl. Phys. Lett. 48, 183 (1986).

${ }^{6}$ D. Moses, J. Wang, A. J. Heeger, N. Kirova, and S. Brazovski, Proc. Natl. Acad. Sci. USA 98, 13496 (2001).

${ }^{7}$ J. A. Barker, C. M. Ramsdale, and N. C. Greenham, Phys. Rev. B 67, 075205 (2003).

${ }^{8}$ L. J. A. Koster, E. C. P. Smits, V. D. Mihailetchi, and P. W. M. Blom, Phys. Rev. B 72, 085205 (2005).

${ }^{9}$ T. Kirchartz, B. E. Pieters, K. Taretto, and U. Rau, J. Appl. Phys. 104, 094513 (2008).

${ }^{10}$ C. Deibel, A. Wagenpfahl, and V. Dyakonov, Phys. Status Solidi RRL 2, 175 (2008).
}

${ }^{11}$ R. Häusermann, E. Knapp, M. Moos, N. A. Reinke, T. Flatz, and B. Ruhstaller, J. Appl. Phys. 106, 104507 (2009).

${ }^{12}$ I. A. Howard, R. Mauer, M. Meister, and F. Laquai, J. Am. Chem. Soc. 132, 14866 (2010).

${ }^{13}$ F. Etzold, I. A. Howard, R. Mauer, M. Meister, T. D. Kim, K. S. Lee, N. S. Baek, and F. Laquai, J. Am. Chem. Soc. 133, 9469 (2011).

${ }^{14}$ M. Weiter, H. Bässler, V. Gulbinas, and U. Scherf, Chem. Phys. Lett. 379, 177 (2003).

${ }^{15}$ C. L. Braun, J. Chem. Phys. 80, 4157 (1984).

${ }^{16}$ W. J. Grzegorczyk, T. J. Savenije, T. E. Dykstra, J. Piris, J. M. Schins, and L. D. A. Siebbeles, J. Phys. Chem. C 114, 5182 (2010).

${ }^{17}$ D. Veldman, O. Ipek, S. C. J. Meskers, J. Sweelssen, M. M. Koetse, S. C. Veenstra, J. M. Kroon, S. S. Bavel, J. Loos, and R. A. J. Janssen, J. Am. Chem. Soc. 130, 7721 (2008).

${ }^{18}$ V. D. Mihailetchi, L. J. A. Koster, J. C. Hummelen, and P. W. M. Blom, Phys. Rev. Lett. 93, 216601 (2004).

${ }^{19}$ C. Deibel, T. Strobel, and V. Dyakonov, Phys. Rev. Lett. 103, 036402 (2009) 
${ }^{20}$ M. M. Mandoc, W. Veurman, L. J. A. Koster, B. de Boer, and P. W. M. Blom, Adv. Funct. Mater. 17, 2167 (2007).

${ }^{21}$ S. H. Park, A. Roy, S. Beaupré, S. Cho, N. Coates, J. S. Moon, D. Moses, M. Leclerc, K. Lee, and A. J. Heeger, Nat. Photonics 3, 297 (2009).

${ }^{22}$ M. Limpinsel, A. Wagenpfahl, M. Mingebach, C. Deibel, and V. Dyakonov, Phys. Rev. B 81, 085203 (2010).

${ }^{23}$ R. Mauer, I. A. Howard, and F. Laquai, J. Phys. Chem. Lett. 1, 3500 (2010).

${ }^{24}$ T. Kirchartz, B. E. Pieters, J. Kirkpatrick, U. Rau, and J. Nelson, Phys. Rev. B 83, 115209 (2011).

${ }^{25}$ S. Schäfer, A. Petersen, T. A. Wagner, R. Kniprath, D. Lingenfelser, A. Zen, T. Kirchartz, B. Zimmermann, U. Würfel, X. Feng et al., Phys. Rev. B 83, 165311 (2011).

${ }^{26}$ R. C. I. MacKenzie, T. Kirchartz, G. F. A. Dibb, and J. Nelson, J. Phys. Chem. C 115, 9806 (2011).

${ }^{27}$ A. Petersen, T. Kirchartz, and T. A. Wagner, Phys. Rev. B 85, 045208 (2012).

${ }^{28}$ A. Ojala, A. Petersen, A. Fuchs, R. Lovrincic, C. Pölking, J. Trollmann, J. Hwang, C. Lennartz, H. Reichelt, H. W. Höffken et al., Adv. Funct. Mater. 22, 86 (2012).

${ }^{29}$ C. Uhrich, D. Wynands, S. Olthof, M. K. Riede, K. Leo, S. Sonntag, B. Maennig, and M. Pfeiffer, J. Appl. Phys. 104, 043107 (2008).

${ }^{30}$ R. W. Lof, M. A. van Veenendaal, B. Koopmans, H. T. Jonkman, and G. A. Sawatzky, Phys. Rev. Lett. 68, 3924 (1992).

${ }^{31}$ M. Kröger, S. Hamwi, J. Meyer, T. Riedl, W. Kowalsky, and A. Kahn, Org. Electron. 10, 932 (2009).

${ }^{32}$ D. R. Lide and H. P. R. Frederikse, CRC Handbook of Chemistry and Physics, 1995-1996 (CRC Press, Boca Raton, FL, 1995).

${ }^{33}$ N. M. Kronenberg, M. Deppisch, F. Würthner, H. W. A. Lademann, K. Deing, and K. Meerholz, Chem. Commun. 6489 (2008).

${ }^{34}$ H. Bürckstümmer, N. M. Kronenberg, M. Gsänger, M. Stolte, K. Meerholz, and F. Würthner, J. Mater. Chem. 20, 240 (2010).

${ }^{35}$ N. M. Kronenberg, V. Steinmann, H. Bürckstümmer, J. Hwang, D. Hertel, F. Würthner, and K. Meerholz, Adv. Mater. 22, 4193 (2010).

${ }^{36} \mathrm{P}$. Würfel, Physics of Solar Cells (Wiley-VCH, Weinheim, 2005).

${ }^{37}$ E. A. Katz, D. Faiman, S. M. Tuladhar, J. M. Kroon, M. M. Wienk, T. Fromherz, F. Padinger, C. J. Brabec, and N. S. Sariciftci, J. Appl. Phys. 90, 5343 (2001).

${ }^{38}$ D. Chirvase, Z. Chiguvare, M. Knipper, J. Parisi, V. Dyakonov, and J. C. Hummelen, J. Appl. Phys. 93, 3376 (2003).

${ }^{39}$ G. Garcia-Belmonte, Solar Energy Mater. Solar Cells 94, 2166 (2010).

${ }^{40}$ C. Y. Wu and J. F. Chen, J. Appl. Phys. 53, 3852 (1982).

${ }^{41}$ See Supplemental Material at http://link.aps.org/supplemental/ 10.1103/PhysRevB.85.245208 for drift-diffusion simulations and the application of the Onsager-Braun model.

${ }^{42}$ A. Miller and E. Abrahams, Phys. Rev. 120, 745 (1960).

${ }^{43}$ O. Rubel, S. D. Baranovskii, W. Stolz, and F. Gebhard, Phys. Rev. Lett. 100, 196602 (2008).

${ }^{44}$ A. V. Nenashev, S. D. Baranovskii, M. Wiemer, F. Jansson, R. Österbacka, A. V. Dvurechenskii, and F. Gebhard, Phys. Rev. B 84, 035210 (2011).
${ }^{45}$ W. Liptay, in Advances in Electronic Excitation and Relaxation, edited by E. C. Lim (Academic Press, New York, 1974), pp. 129 229.

${ }^{46}$ A. Horvath, H. Bässler, and G. Weiser, Phys. Status Solidi B 173, 755 (1992).

${ }^{47}$ M. P. Steele, M. L. Blumenfeld, and O. L. A. Monti, J. Phys. Chem. Lett. 1, 2011 (2010).

${ }^{48}$ H. Bässler, Phys. Status Solidi B 175, 15 (1993).

${ }^{49}$ M. Kemerink, J. M. Kramer, H. H. P. Gommans, and R. A. J. Janssen, Appl. Phys. Lett. 88, 192108 (2006).

${ }^{50}$ E. Heinecke, D. Hartmann, and A. Hese, J. Chem. Phys. 118, 113 (2003).

${ }^{51}$ C. She, J. E. McGarrah, S. J. Lee, J. L. Goodman, S. B. T. Nguyen, J. A. G. Williams, and J. T. Hupp, J. Phys. Chem. A 113, 8182 (2009).

${ }^{52}$ F. Würthner, R. Wortmann, and K. Meerholz, Chem. Phys. Chem. 3, 17 (2002).

${ }^{53}$ F. Würthner, G. Archetti, R. Schmidt, and H. G. Kuball, Angew. Chem., Int. Ed. 47, 4529 (2008).

${ }^{54}$ V. I. Arkhipov, P. Heremans, and H. Bässler, Appl. Phys. Lett. 82, 4605 (2003).

${ }^{55}$ S. Verlaak, D. Beljonne, D. Cheyns, C. Rolin, M. Linares, F. Castet, J. Cornil, and P. Heremans, Adv. Funct. Mater. 19, 3809 (2009).

${ }^{56}$ V. I. Arkhipov, E. V. Emelianova, and G. J. Adriaenssens, Phys. Rev. B 64, 125125 (2001).

${ }^{57}$ M. Muntwiler, Q. Yang, W. A. Tisdale, and X. Y. Zhu, Phys. Rev. Lett. 101, 196403 (2008).

${ }^{58}$ T. M. Clarke, A. M. Ballantyne, J. Nelson, D. D. C. Bradley, and J. R. Durrant, Adv. Funct. Mater. 18, 4029 (2008).

${ }^{59}$ H. Ohkita, S. Cook, Y. Astuti, W. Duffy, S. Tierney, W. Zhang, M. Heeney, I. McCulloch, J. Nelson, D. D. C. Bradley et al., J. Am. Chem. Soc. 130, 3030 (2008).

${ }^{60}$ X. Y. Zhu, Q. Yang, and M. Muntwiler, Acc. Chem. Res. 42, 1779 (2009).

${ }^{61}$ S. Shoaee, Z. An, X. Zhang, S. Barlow, S. R. Marder, W. Duffy, M. Heeney, I. McCulloch, and J. R. Durrant, Chem. Commun. 5445 (2009).

${ }^{62}$ T. M. Clarke, A. M. Ballantyne, S. Tierney, M. Heeney, W. Duffy, I. McCulloch, J. Nelson, and J. R. Durrant, J. Phys. Chem. C 114, 8068 (2010).

${ }^{63}$ C. G. Shuttle, R. Hamilton, B. C. O'Regan, J. Nelson, and J. R. Durrant, Proc. Natl. Acad. Sci. USA 107, 16448 (2010).

${ }^{64}$ R. A. Street, S. Cowan, and A. J. Heeger, Phys. Rev. B 82, 121301 (2010).

${ }^{65}$ U. Albrecht and H. Bässler, Chem. Phys. Lett. 235, 389 (1995).

${ }^{66}$ S. Barth, H. Bässler, U. Scherf, and K. Müllen, Chem. Phys. Lett. 288, 147 (1998).

${ }^{67}$ D. Hertel, U. Scherf, and H. Bässler, Adv. Mater. 10, 1119 (1998).

${ }^{68}$ V. I. Arkhipov, E. V. Emelianova, and H. Bässler, Phys. Rev. Lett. 82, 1321 (1999).

${ }^{69}$ V. I. Arkhipov, E. V. Emelianova, S. Barth, and H. Bässler, Phys. Rev. B 61, 8207 (2000).

${ }^{70}$ D. M. Basko and E. M. Conwell, Phys. Rev. B 66, 155210 (2002). 